\title{
Updates in Management of Hypertensive Disorders of Pregnancy
}

\author{
Abanoub Gabra ${ }^{1 *}$ and Mariam Gabra ${ }^{2}$ \\ ${ }^{1}$ Department of Obstetrics and Gynecology, Assuit University, Egypt \\ ${ }^{2}$ Hillsborough Community College, Florida, USA
}

*Corresponding author: Abanoub Gabra, Department of Obstetrics and Gynecology, Assuit University, Egypt.

Received Date: May 20, 2019

Published Date: May 28, 2019

\begin{abstract}
Hypertensive disorders of pregnancy (HDP) were discovered a long time ago but our knowledge about their etiopathogenesis are still limited. These disorders can affect many pregnant women all over the world putting them, their families and their countries under significant health burden. The main goal of all research efforts regarding treatment of HDP is how to prevent highly morbid complications and mortality of HDP that may affect both mother and fetus. Delivery is the main line of treatment for severe cases, but we have to put in our mind risk of prematurity as a possible outcome in case of termination besides the possibility of incidence of complications like eclampsia and hypertensive emergency postnatally. Researchers believed that studies working on prevention would result in major improvement of overall women care worldwide, and screening will definitely allow prompt starting therapy for those patients and prevent further sequences. Recently many guidelines support and recommend Aspirin $81 \mathrm{mg}$ for the prevention of preeclampsia in high-risk patients using evidence-based data about the time and indications of its intake. Great efforts have been contributed to developing evidence-based guidelines to enhance our strategies to deal with these disorders. Reviewing, pooling and comparing these guidelines would help us to refine our questions that may need to be answered by further studies. Trials confirmed that application of standardized strategies for treating HDP significantly improves both maternal and fetal outcomes. In this article, we are going to review recent updates and guidelines about the treatment of HDP and provide references for your further readings.
\end{abstract}

Keywords: Hypertension; Pregnancy; Management; Guidelines

\section{Introduction}

Interestingly, Hypertensive disorders of pregnancy (HDP) were discovered two thousand years ago but our knowledge and data about their nature and pathogenesis are still limited [1]. These disorders can affect many pregnant women all over the world putting them, their families and their countries under significant health burden. For instance, in USA, 240.000 pregnant suffer HDP which equal to $6-12 \%$ of all pregnancies [2] and this accounts for $10 \%$ of maternal morality being the second common cause of maternal death after postpartum hemorrhage [3]. Moreover, HDP may cause intrauterine fetal death, intrauterine growth restriction and prematurity [4]. Higher prevalence of HDP was found in African Americans, obese, patients with renal disease, chronic hypertensive patients, patients with autoimmune diseases, multiple pregnancy, diabetics and in pregnant women $>35$ years old or less than twenty $[5,6]$. Moreover, when we imagine the size of that problem in developing countries, we realize that it is a real disaster affecting families worldwide [7].

Many societies try to classify HDP in spite of presence of an overlap between these classes and subgroups under each category [1]. According to The American College of Obstetricians and Gynecologists (ACOG) and the National High Blood Pressure Education Program Working group on High blood pressure in pregnancy, HDP is classified into chronic hypertension, gestational hypertension, preeclampsia-eclampsia, and preeclampsiaeclampsia superimposed on chronic hypertension [7]. There is difference in hemodynamic and neurohormonal profile of each category [8]. The main goal of all research efforts in treating HDP is how to prevent highly morbid complications and mortality of HDP that may affect both mother and fetus [9]. Mothers with HDP are at risk of renal and liver failure as part of multisystem nature 
of the disease that is not understood completely. Besides that, coagulopathies increase her risk of postpartum hemorrhage which is the leading cause of maternal mortality worldwide [10]. Indeed, HDP increase risk of cardiovascular and cerebrovascular event in the lifetime and its mortality through their systematic metabolic and vascular abnormal changes [11]. With the growing concept of the 4th-trimester care, HDP remain a cornerstone in the postnatal care which includes measuring BP and patient's awareness of symptoms of that disease till 4-6 weeks to anticipate any complications that may threaten women health [1].

Because of all those complications, researchers believed that studies working on prevention would result in major improvement of overall women care worldwide [11,12], and screening will definitely allow prompt starting therapy for those patients. In this article, we are going to review recent updates and guidelines about the treatment of HDP and provide references for your further readings.

\section{Management}

Great efforts have been contributed to developing evidencebased guidelines to enhance our strategies to deal with these disorders. Reviewing, pooling and comparing these guidelines would help us to refine our questions that may need to be answered by further studies. Trials confirmed that application of standardized strategy for treating HDP improves so much both maternal and fetal outcome $[13,14]$

\section{Chronic hypertension}

Chronic hypertension affects about 5\% of pregnancies and increases the risk of both fetal and maternal morbidity [15]. It is defined as systolic BP of 140 or more Or diastolic BP of 90 or more Or both on 2 separate occasions at least 4 hours apart [16]. This condition is diagnosed at or before 20 gestational weeks or already documented before pregnancy [17], however, ACOG suggest that gestational hypertension or early onset preeclampsia should be considered if 1st trimester BP measures are within normal range [18]. Mostly clinicians attribute this condition to essential hypertension, but they should consider secondary causes in case of young aged onset and refractoriness to therapy [15].

Regarding management, chronic hypertension should be managed because of its lifelong end-organ damaging effects but pregnancy is a short time event and data suggest fetal risk with excessive low BP and placental hypoperfusion [19] so pharmacological treatment is recommended when BP equal or is greater than $150 / 100 \mathrm{mmHg}$ [16]. But other researchers recommend the goal of below 140/90 when patient has multiple risk factors that increase risk of maternal mortality and morbidity [15].

Complete metabolic panel should be done, and patient should be counseled about daily BP monitoring with log and how to use automated devices, we can use video materials like the educational video of American Medical Association (AMA) and American Heart Association (AHA). She should be also counseled in both antepartum and postpartum care visits about symptoms of severe preeclampsia and imminent eclampsia [15]. Moderate exercise is encouraged but weight loss and extreme low salt diet are not recommended [19]. First lines drugs are alpha-methyldopa, nifedipine and labetalol and drugs affecting the renin-angiotensin system should be avoided because of their well know teratogenic effect [19]. Diuretics are also not recommended because it aggravates hemoconcentration of preeclampsia which may predispose to clots [20].

According to ACOG, Fetal wellbeing should be assessed by ultrasound and umbilical artery Doppler should be done to assess placental perfusion in case of abnormal US findings like IUGR and oligohydramnios [19]. NICE recommends fetal assessment by US and umbilical artery Doppler at 28-30 weeks and at 32-34 weeks. if results are normal, clinicians should not repeat them unless clinically indicated [20].

In uncomplicated patients of chronic hypertension, delivery is not recommended before 38 weeks but progression of the disease to preeclampsia and eclampsia affect decision making regarding time of delivery [19].

\section{Gestational hypertension and Preeclampsia without severe features}

Gestational hypertension is defined as elevated BP above 140/90 mmHg after 20 weeks at two separate occasions 6 hours apart in absence of features of severe preeclampsia [21]. Severe gestational hypertension is defined by sustained elevated BP at more than or equal $160 / 110 \mathrm{mmHg}$ [22]. It is the most common cause of high BP in pregnancy $[1,22]$. Some authors recommend the term "pregnancy-induced hypertension" to describe this condition that may include preeclampsia as well [2]. This condition should be distinguished from chronic hypertension [22] and transient gestational hypertension. The return of BP to normal after 42 days postpartum means that it is gestational hypertension, this indicates the importance of postpartum care for those patients and the relative accuracy of diagnosis till that time [5]. Presence of proteinuria indicates its progression to preeclampsia [19]. Those cases may develop severe preeclampsia or eclampsia with progression of pregnancy especially with elevated uric acid and with onset at less than 34 weeks [23]. some cases with mild disease and its onset at or beyond 37 weeks have a similar outcome to normotensive cases [22].

Gestational hypertension and preeclampsia without severe features are managed by the same strategy, control of hypertension and expectant management are the main concepts in this strategy [16]. Prenatal care should include BP measurement and proteinuria once weekly at office besides assessment of platelet count and liver enzymes are suggested weekly in order to detect progression to severe preeclampsia $[19,22]$. As well, patients should be counseled about symptoms of severe preeclampsia and seeking ER as rapid as they can [22]. Bed rest is no longer recommended especially with raising concerns of thromboembolism [19,22]. Because of risk of fetoplacental insufficiency, fetal monitoring should be done twice 
weekly in case of preeclampsia or once weekly in case of gestational hypertension by US (include Amniotic Fluid Index) and Non-Stress Test besides daily count of fetal movements [19,24]. Fetal monitoring in form of Non-Stress Test and Biophysical Profile [22] should be done till 34 weeks and it should not be repeated after excepting if clinically indicated (20). Pharmacological therapies are reserved to patients with BP more than 160/110 [7] and the same drugs as chronic hypertension with well documented fetal safety [19]. This concept is applied till 37-39 weeks or more in presence adequate resources in a nearby maternal-fetal unit $[18,20,23]$. Magnesium sulfate is not recommended for seizure prophylaxis because of its low incidence in gestational hypertension [1]. In presence of rupture of membranes, abnormal fetal monitoring [16] or ultrasonographic findings of fetal growth restriction confirmed by Doppler, delivery is indicated at 34 weeks $[19,20]$. But in presence of any maternal or fetal instability, delivery is recommended as soon as possible after stabilization of general condition [19].

\section{Preeclampsia with severe features / eclampsia}

SOMANZ defines preeclampsia with severe features as a unique condition of pregnancy with multisystemic effects involving liver, kidney and other hematological parameters [17]. ACOG criteria for diagnosis include new onset hypertension after 20 weeks associated with new onset proteinuria $(>300 \mathrm{mg} / 24$ hours urine collection) [19]. In absence of proteinuria can be made upon presence of gestational hypertension plus any of the following: low platelet count less than $100.000 / c c$, creatinine level more than 1.1 ( double the baseline creatinine level in absence of other renal problem), raised liver enzymes ( double baseline), pulmonary edema, visual or cerebral symptoms [18]. ACOG recommends that decision of delivery should be made based neither on the severity of proteinuria nor on the change in the amount of proteinuria [18]. As a result of our limited knowledge about etiopathogenesis of preeclampsia, we should expand our management to involve preconception care of high-risk women or patients with positive personal history until 4th-trimester care postnatally [25].

The rationale of management of those patients focused on riskbenefit balance of termination of pregnancy to avoid maternal or fetal complications or cautious expectant management to avoid neonatal risks of prematurity [26]. the decision should be made based on severity of the disease and gestational age because initial presentation is not a reliable tool to predict the course of progression of the disease [11]. When expectant management is considered till 34 weeks, ACOG recommends steroid administration to enhance fetal lung maturity [18] and close monitoring for signs and symptoms of severity on both mother and fetus should be considered. Reviewers of Cochrane trials conclude that there is a lack of sufficient evidence in order to make a clinical guidance for bed rest and it should not be routinely recommended [27]. Most guidelines agree that termination should be done at or beyond 37 weeks, it could be also done at or beyond 34 weeks in presence of IUGR, oligohydramnios, rupture of membranes, progressive labor and biophysical profile of $6 / 10$ or less $[17,18,23,26]$. But in presence of any fetal or maternal instability, delivery is indicated regardless of gestational age (19)(28) because delivery has a very beneficial effect on preventing serious maternal complications [27]. As control of BP alone does not prevent seizures, Magnesium sulfate is a cornerstone in the prevention of eclampsia [26]. It should be administered prenatally, at labor and at least 24 hours postnatally [11]. Researchers suggest that it may contribute to slowing neuromuscular conduction and increase seizure threshold [27]. Special protocols are used for patients with renal insufficiency in addition to regular monitoring of serum $\mathrm{Mg}$ level and watchful observation of signs of Mg toxicity [11]. Pharmacological treatment of cases with BP above 160/100 has been shown to decrease the incidence of a cerebral vascular incident on those patients [11]. NICE guidelines support labetalol as combined alpha and beta blocker as the first line of treatment [20]. ACOG and SOGC recommend labetalol, hydralazine, and nifedipine as first-line agents [29]. ACOG recommends that mode of delivery should be individualized based on fetal presentation, gestational age, cervical status, and maternalfetal stability and it does not need to be generalized as cesarean section [18]. Combined oxytocin and ergometrine use should be avoided in managing the third stage of labor as ergots cause more elevation of BP in those patients [27].

\section{Emergency Therapy}

Acute onset of severe hypertension is defined as BP higher than $170 / 110$ and it is considered persistent if remains the same 5-15 minutes later [30]. it is associated with a higher incidence of cerebrovascular stroke and eclampsia in preeclamptic patients [14] so proper antihypertensives should be administrated within 15-30 minutes of confirmed severe hypertension [14]. This condition requires urgent antihypertensive medications and close observation of both mother and her fetus [14]. ACOG and the National heart, lung and blood institute recommend that immediate release oral Nifedipine, intravenous labetalol, and hydralazine are the first lines of treatment for hypertensive emergency either during pregnancy or postnatally $[14,31]$. In a randomized doubleblinded clinical trial of both oral nifedipine and IV labetalol, both found to be effective in this condition [32] but oral nifedipine was found to be rapidly effective, has a longer duration of action and associated with improved urine output and less side effect $[33,34]$. Dropping of BP with Oral nifedipine is associated with no change in fetal heart rate [35] nor in fetal and uteroplacental Doppler indices [36], although it is associated with a drop of mean blood pressure and systemic vascular resistance in addition to enhancement of cardiac index which is an accurate parameter of cardiac output [37]. Co-administration of nifedipine and magnesium sulfate do not increase incidence of toxicity and side effects of Mg sulfate [38]. Limited evidence suggests the effectiveness of nicardipine in this condition with safe maternal and fetal profile $[39,40]$. In refractory case, clinicians should seek consultation of feto-maternal or intensive care subspecialists for lines to control this condition [14].

\section{Role of Preventive Medicine}

Strong evidence of the role of low dose aspirin $(<81 \mathrm{mg}$ ) causes many societies concerned about women health to admit its benefits 
in preeclampsia prevention in high risk pregnant women. ACOG, USPSTF, WHO, NICE and AAFP recommend its intake after 1st trimester at 12-16 weeks [4]. USPSTF recommends it in presence of one or more of high-risk factors like history of preeclampsia, multiple gestation, kidney disease, autoimmune disease like antiphospholipid syndrome, type 1 or type 2 diabetes, and chronic hypertension or more than one of several moderate-risk factors like primigravidity, maternal age of 35 years or older, a body mass index greater than 30 , family history of preeclampsia, sociodemographic characteristics, and personal history factors [6] In absence of highrisk factors for preeclampsia, current evidence-based data does not support its use for the prevention of preeclampsia, IUGR, IUFD, or prematurity [6].

Evidence-based guidelines support Calcium supplements as a preventive tool against preeclampsia in societies with low calcium intake [18]. High dose Methylprednisolone was not found to be effective to prevent drop of platelets count in preeclamptic patient with count 150.000 unit/cc or keep it above 100.000 unit/ cc [41]. 5-day postpartum course of Torsemide was not found to be effective in prevention of persistent postpartum preeclampsia [42]. Effective prenatal care and frequent antenatal visits are associated with improved outcomes of HDP regarding both maternal and fetal health [43]. USPSTF recommends screening for preeclampsia by frequent measurement of blood pressure through antenatal care [44]. ACOG recommends BP measure and detailed medical history to evaluate risk factors [18]. NICE and SOGC still recommend urinalysis for proteinuria in addition to BP measurement $[44,45]$.

\section{Summary}

Hypertensive disorders of pregnancy (HDP) were diseases of a long time ago but our knowledge about their etiopathogenesis are still in need of more researches. These disorders can affect many pregnant women all over the world putting them, their families and their countries under significant health burden. The main goal of research efforts is how to prevent highly morbid complications and mortality of HDP that may affect both mother and fetus. Delivery remains the main line of treatment for severe cases indicating the role of fetoplacental unit in the disease, but we must consider the risk of prematurity as a possible outcome in case of termination besides the possibility of incidence of complications like eclampsia and hypertensive emergency postnatally. Researchers believed that studies working on prevention would result in impressive improvement of overall women care worldwide, and screening will allow prompt starting of treatment for those patients and prevent further sequences. Recently many guidelines support and recommend Aspirin 81mg for the prevention of preeclampsia in high-risk patients using evidence-based data about the time and indications of its intake. Great efforts have been contributed to developing evidence-based guidelines to enhance our strategies to deal with these disorders. Reviewing, pooling and comparing these guidelines would help us to refine our questions that may need to be answered by further studies. Trials confirmed that application of standardized strategies improves both maternal and fetal outcomes.

\section{Acknowledgement}

None.

\section{Conflict of Interest}

No conflict of interest.

\section{References}

1. Rosser ML, Katz NT (2013) Preeclampsia: an obstetrician's perspective. Adv Chronic Kidney Dis 20(3): 287-296.

2. Paul E, Marik (2016) Hypertensive disorders of pregnancy. Am Fam Physician 93(2):121-127.

3. (2013) CMQCC. Improving Health Care Response to Preeclampsia: A California Quality Improvement Toolkit. 1-158.

4. LeFevre ML (2014) Low-dose aspirin use for the prevention of morbidity and mortality from preeclampsia: U.S. Preventive Services Task Force recommendation statement. Ann Intern Med 161(11): 819-826.

5. Tiina Podymow, Phyllis August, Amanda R Vest, Leslie S Cho, Amanda R Vest, et al. (2012) Hypertension in Pregnancy. Cardiovasc Ther A Companion to Braunwald's Hear Dis Fourth14(2): 521-528.

6. (2018) ACOG Committee Opinion Low-Dose Aspirin Use During Pregnancy. Obstet Gynecol 132(1): e44-e52.

7. Jim B, Sharma S, Kebede T, Acharya A (2010) Hypertension in pregnancy: A comprehensive update. Cardiol Rev 18(4): 178-189.

8. Borghi C, Cicero AF, Degli Esposti D, Immordino V, Bacchelli S, et al. (2011) Hemodynamic and neurohumoral profile in patients with different types of hypertension in pregnancy. Intern Emerg Med 6(3): 227-234.

9. Kattah AG, Garovic VD (2013) The management of hypertension in pregnancy. Adv Chronic Kidney Dis 20(3): 229-239.

10. Sibai BM (2012) Etiology and management of postpartum hypertensionpreeclampsia. Am J Obstet Gynecol 206(6): 470-475.

11. Wagner SJ, Barac S, Garovic VD (2007) Hypertensive pregnancy disorders: current concepts. J Clin Hypertens (Greenwich) 9(7): 560566.

12. Huda SS, Freeman DJ, Nelson SM (2009) Short- and long-term strategies for the management of hypertensive disorders of pregnancy. Expert Rev Cardiovasc Ther 7(12): 1581-1594.

13. Dadelszen P Von, Sawchuck D, Mcmaster R (2010) The Active Implementation of Pregnancy hypertension guidelines in British Columbia. Obstet Gynecol 116(3): 659-666.

14. (2017) Emergent Therapy for Acute-Onset, Severe Hypertension During Pregnancy and the Postpartum Period, Committee on Obstetric Practice, ACOG.

15. Ankumah NE, Sibai BM (2017) Chronic Hypertension in pregnancy: diagnosis and management. Clin Obstet Gynecol 60(1): 206-214.

16. Leeman Lawrence lee t, dresang FP (2016) Hypertensive disorders of pregnancy. Am Acad Fam Physicians 87(2):194-198.

17. Lowe SA, Brown MA, Brown M a, Dekker GA, Dekker G a, Gatt S, et al. (2009) Guidelines for the management of hypertensive disorders of pregnancy SOMANZ 2008. Aust N Z J Obstet Gynaecol 49: 242-246.

18. Roberts James M, Druzin Maurice, August Phyllis A, Gaiser Robert R, Bakris George, et al. (2013) Hypertension in pregnancy, ACOG guidelines. pp.1-100.

19. Roberts James M, Druzin Maurice, August Phyllis A, Gaiser Robert R, Bakris George, et al. (2013) Report of the American College of Obstetricians and Gynecologists' Task Force on Hypertension in Pregnancy 122(5): 1-100.

20. Visintin C, Mugglestone MA, Almerie MQ, Nherera LM, James D (2010) Management of hypertensive disorders during pregnancy: Summary of NICE guidance. BMJ 341(7771): 499-501. 
21. Amelia LM, Sutton, Lorie MH, Alan TN, Tita P (2018) Hypertensive Disorders in Pregnancy. Obstet Gynecol Clin North Am. 45(2):333-47.

22. Sibai BM. Diagnosis and Management of Gestational Hypertension and Preeclampsia. ACOG, Elsevier. 1996;41(8):67-78.

23. Brown MA, Magee LA, Kenny LC, Karumanchi SA, McCarthy FP, Saito S, et al. (2018) Hypertensive disorders of pregnancy: ISSHP classification, diagnosis, and management recommendations for international practice. Hypertension 72(1): 24-43.

24. Deak TM, Moskovitz JB (2012) Hypertension and Pregnancy. Emerg Med Clin North Am 30(4): 903-917

25. Phipps E, Prasanna D, Brima W, Jim B (2016) Preeclampsia: Updates in pathogenesis, definitions, and guidelines. Clin J Am Soc Nephrol 11(6): 1102-1113.

26. Dhariwal NK, Lynde GC (2017) Update in the Management of Patients with Preeclampsia. Anesthesiol Clin 35(1): 95-106.

27. Amanda RV, Leslie SC (2014) Hypertension in Pregnancy. Cardiovasc Ther A Companion to Braunwald's Hear Dis ( $4^{\text {th }}$ edn): 521-528.

28. Cicero AF, Degli Esposti D, Immordino V, Morbini M, Baronio C, et al. (2015) Independent Determinants of Maternal and Fetal Outcomes in a Sample of Pregnant Outpatients with Normal Blood Pressure, Chronic Hypertension, Gestational Hypertension, and Preeclampsia 17(10): 777-782.

29. Amanda RV, Leslie SC (2012) Hypertension in Pregnancy. Cardiovasc Ther A Companion to Braunwald's Hear Dis 30(3): 521-528.

30. Magee LA, von Dadelszen P (2009) The Management of Severe Hypertension. Semin Perinatol 33(3): 138-142.

31. The Seventh Report of the Joint National Committee on Prevention, Detection, Evaluation, and Treatment of High Blood Pressure, Complete Report the Seventh Report of the Joint National Committee on Prevention, Detection, Evaluation, and Treatment of High Bl.

32. IA Raheem \& R Saaid\& SZ Omar, PC Tan (2015) Oral nifedipine versus intravenous labetalol in hypertensive emergencies of pregnancy: A randomised trial. Res J Pharm Biol Chem Sci 6(2): 1673-1681.

33. Rezaei Z, Sharbaf FR, Pourmojieb M, Youefzadeh-Fard Y, Motevalian M, et al. (2011) Comparison of the efficacy of nifedipine and hydralazine in hypertensive crisis in pregnancy. Acta Med Iran 49(11): 701-706.

34. Vermillion ST, Scardo JA, Newman RB, Chauhan SP (1999) A randomized, double-blind trial of oral nifedipine and intravenous labetalol in hypertensive emergencies of pregnancy. Am J Obstet Gynecol 181(4): 858-861.

35. Samuel Lurie, Katerina Fenakel, Adi Friedman M (1990) Effect of nifedipine on fetal heart rate in pre-eclamptic patients. Am J Perinatol.

36. Moretti MM, Fairlie FM, Akl S, Khoury AD, Sibai BM (1990) The effect of nifedipine therapy on fetal and placental Doppler waveforms in preeclampsia remote from term. Am J Obstet Gynecol 163(6 Pt 1): 18441848

37. Scardo JA, Vermillion ST, Hogg BB, Newman RB (1996) Hemodynamic effects of oral nifedipine in preeclamptic hypertensive emergencies. Am J Obstet Gynecol 175(2): 336-340.

38. Magee LA, Miremadi S, Li J, Cheng C, Ensom MH, et al. (2005) Therapy with both magnesium sulfate and nifedipine does not increase the risk of serious magnesium-related maternal side effects in women with preeclampsia. Am J Obstet Gynecol 193(1): 153-163.

39. Nij Bijvank SW, Duvekot JJ. (2010) Nicardipine for the treatment of severe hypertension in pregnancy: A review of the literature. Obstet Gynecol Surv 65(5): 341-347.

40. Vadhera RB, Pacheco LD, Hankins GD (2009) Acute Antihypertensive Therapy in Pregnancy-Induced Hypertension: Is Nicardipine the Answer? Am J Perinatol 26(7): 495-499.

41. Pourrat O, Dorey M, Ragot S, De Hauteclocque A, Deruelle P, et al. (2016) High-dose methylprednisolone to prevent platelet decline in preeclampsia. Obstet Gynecol 128(1): 153-158.

42. Viteri OA, Alrais MA, Pedroza C, Hutchinson M, Chauhan SP, et al. (2018) Torsemide for Prevention of Persistent Postpartum Hypertension in Women with Preeclampsia. Obstet Gynecol 132(5): 1185-1191.

43. Witlin AG, Sibai BM (1997) Hypertension in pregnancy: current concepts of preeclampsia. Annu Rev Med 48(1): 115-127.

44. Bibbins-Domingo K, Grossman DC, Curry SJ, Barry MJ, Davidson KW, et al. (2015) Screening for Preeclampsia US Preventive Services Task Force Recommendation Statement. JAMA 317(16): 1661-1667.

45. Magee LA, Pels A, Helewa M, Rey E, von Dadelszen P, et al. (2014) Diagnosis, Evaluation, and Management of the Hypertensive Disorders of Pregnancy: Executive Summary. J Obstet Gynaecol Canada 36(5): 416-438. 